\title{
Passivity Check of $S$-Parameter Descriptor Systems via $S$-Parameter Generalized Hamiltonian Methods
}

\author{
Zheng Zhang, Student Member, IEEE, and Ngai Wong, Member, IEEE
}

\begin{abstract}
This paper extends the generalized Hamiltonian method (GHM) (Zhang et al., 2009; Zhang and Wong, 2010) and its half-size variant (HGHM) (Zhang and Wong, 2010) to their $S$-parameter counterparts (called S-GHM and S-HGHM, respectively), for testing the passivity of $S$-parameter descriptor-form models widely used in high-speed circuit and electromagnetic simulations. The proposed methods are capable of accurately detecting the possible nonpassive regions of descriptor-form models with either scattering or hybrid (impedance or admittance) transfer matrices. Their effectiveness and accuracy are verified with several practical examples. The S-GHM and S-HGHM methods presented here provide a foundation for the passivity enforcement of $S$-parameter descriptor systems.
\end{abstract}

Index Terms-Descriptor system (DS), $S$-parameter generalized Hamiltonian method (S-GHM), $S$-parameter half-size GHM (S-HGHM), system passivity.

\section{INTRODUCTION}

A $\mathrm{S}$ a superset of nonsingular or regular systems, descriptor (or singular) systems (DSs) [3], [4] are capable of describing a much larger variety of physical models. In linear circuit simulation, modified nodal analysis (MNA) formulations of $R L C$ networks (such as interconnect and power grid models [5], [6]) are DSs. In nonlinear circuit analysis, the piece-wise linearization procedure also generates descriptor-form models [7]. Even the piece-wise polynomial representation of an analog/RF circuit can be treated as the interconnection of several coupled DSs [8]. In electromagnetic (EM) modeling of devices, connectors and on-chip passives [9]-[11], DS is also widely used: discretized EM equations are usually of DS format. Compared with regular systems, DSs can provide more information, such as the possible impulsive port response. For some impulse-free physical systems, although the port responses can be characterized by nonsingular systems, converting the more natural DSs to regular ones can be extremely expensive and sometimes numerically unstable [12]. In many cases, it is also desirable to keep the physical models in the more natural DS format to facilitate fast computation through possible utilization of matrix structures and sparsity.

Manuscript received January 01, 2010; revised March 30, 2010; accepted June 03, 2010. Date of publication July 19, 2010; date of current version January 07,2011 . This work was supported in part by Hong Kong Research Grants Council under Project 717407E and Project 718509E, and in part by the University Research Committee of The University of Hong Kong. This work was recommended for publication by Associate Editor J. Tan upon evaluation of the reviewers comments.

The authors are with the Department of Electrical and Electronic Engineering, The University of Hong Kong, Hong Kong (e-mail: zzhang@eee.hku.hk; nwong@eee.hku.hk).

Color versions of one or more of the figures in this paper are available online at http://ieeexplore.ieee.org.

Digital Object Identifier 10.1109/TADVP.2010.2052807
Normally, the sizes of the DS models in circuit and EM simulations are very large, rendering direct simulation prohibitively time-consuming. Therefore, model order reduction (MOR) techniques [5], [11]-[14] have been widely used to approximate the original models by much smaller ones. On the other hand, from the black-box perspective, given a set of measured input-output frequency data, a macromodel can also be built to approximate the original system. Physically, these measured data may represent the admittance, impedance or scattering parameters at a set of frequency points. In high-frequency applications, scattering parameters are more commonly used due to their relative ease of measurement. Using such data fitting techniques as vector fitting [15], standard state-space macromodels can be constructed. Recently, the Loewner matrix interpolation technique [16], [17] has been advocated to fit measured/simulated data of electronic circuits/systems to produce the corresponding DS. Such framework is superior to the traditional vector fitting approach in the sense that no manual pole initialization is needed and that the optimal model order can be automatically extracted.

Using either the original models, the reduced models from MOR, or the macromodels from data fitting, to guarantee globally stable circuit or system simulations, these models are usually required to be passive, because a system consisting of interconnected passive subsystems is guaranteed to be stable. In contrast, the interconnection of stable but nonpassive subblocks may result in unstable responses depending on the terminations. If the obtained models are nonpassive, enforcement techniques may be applied to mitigate or compensate system passivity [18]-[22], where passivity verification needs to be performed in advance to locate the nonpassive frequency intervals. For standard state-space models, frequency sweeping tests [18], [19] and the more reliable Hamiltonian methods [20]-[22] are widely used. To accelerate computation, half-size singularity tests have been developed for symmetric cases [23], [24], which bring about an $8 \times$ speedup versus traditional full-size Hamiltonian methods.

For DS models, several algebraic passivity tests have been proposed for hybrid (admittance or impedance) cases [25]-[30]. However, their expensive computation [25] and requirements of minimal or admissible [26]-[30] realizations render them impractical for general DS models. Moreover, due to their inability to locate DS nonpassive regions, they are not good choices for passivity enforcement flows. To address this problem, the frequency sweeping technique has been extended to DS cases [31], [32]. However, due to the sampling nature of frequency sweeping, no guarantee can be made for the complete identification of all nonpassive regions. To this end, the recently proposed GHM/HGHM test [1], [2] delivers as high 
a numerical accuracy (of locating all nonpassive frequency intervals) for DSs as that of Hamiltonian methods [20]-[24] for regular state spaces.

In the context of $S$-parameter DSs, passivity verification is still not well addressed. Although the extended bounded-real lemma [33] and GARE-based method [34] have been proposed for passivity check, no reliable technique exists for the nonpassive region identification. Due to the lack of reliable $S$-parameter DS passivity verification algorithms, passivity enforcement can not be performed for nonpassive models at present. Motivated by this demand, this paper extends the GHM and HGHM theories to their $S$-parameter counterparts called the S-GHM and S-HGHM, respectively, to verify the passivity of the physical models in circuit or EM simulation. A preliminary version of this work, without the half-size implementation, has been reported in [35]. Although this work focuses on $S$-parameter DS passivity test, a reliable passivity compensation approach can be straightforwardly developed based on the theories presented here, which would be documented in our future reports very soon.

\section{BACKGROUND OF PASSIVITY CHECK}

Throughout this paper, the superscripts ${ }^{T}$ and ${ }^{*}$ denote transpose and conjugate transpose (Hermitian) operations, respectively. For a general matrix $X, \sigma(X)$ represents the set of singular values in descending magnitudes, and $\sigma_{i}(X)$ denotes the $i$ th singular value. The set $\lambda(X, Y)$ means the generalized eigenvalues of the matrix pencil $(X, Y)$ [i.e., $\operatorname{det}(X-a Y)=0$ if $a(\in \mathbb{C}) \in \lambda(X, Y)]$. The set $\lambda(X, I)$ is sometimes abbreviated as $\lambda(X)$, where $I$ is an identity matrix.

We consider the continuous linear time-invariant (LTI) DS model $\Sigma:(E, A, B, C, D)$, with the state-space equations

$$
E \dot{x}=A x+B u, \quad y=C x+D u .
$$

Here $E, A \in \mathbb{R}^{n \times n}, B \in \mathbb{R}^{n \times m}, C \in \mathbb{R}^{m \times n}, D \in \mathbb{R}^{m \times m}$, and $x \in \mathbb{R}^{n}$ represents the state variables. In this DS, $\operatorname{rank}(E) \leq$ $n$ and the matrix pencil $(A, E)$ is assumed to be regular, i.e., $\operatorname{det}(A-s E) \neq 0$ for some $s \in \mathbb{C}$. If $E$ is full-rank (or invertible), the DS reduces to a regular system and then can be converted to a standard state-space equation by absorbing $E^{-1}$ into $A$ and $B$.

\section{A. Passivity of S-Parameter LTI Systems}

The transfer matrix of system (1) is

$$
S(s)=C(s E-A)^{-1} B+D .
$$

When (1) or (2) represents an $S$-parameter DS, the (strict) passivity is equivalent to the (strict) bounded realness of $S(s)(s=$ $\delta+j \omega$, where $\delta, \omega \in \mathbb{R})$, i.e.,

1) $S(s)$ is analytic on the open right half plane $(\delta>0)$;

2) $I-S^{*}(j \omega) S(j \omega) \geq 0$ (> for strict bounded realness) for all $\omega$.

Condition 2) implies $\sigma_{1}(S(j \omega)) \leq 1$ (or $<1$ for strict bounded realness), which can be checked by sampling some points (frequencies) along the imaginary axis $s=j \omega$ [18], [19], [31]. However, erroneous results may result if nonpassive regions between sampling points are missed. For standard state-space models $(A, B, C, D)$ [with $E=I$ in (1)], the more reliable Hamiltonian method is preferred. The corresponding $2 n \times 2 n$ Hamiltonian matrix is defined as

$$
\mathcal{M}=\left[\begin{array}{cc}
A-B D^{T} \hat{S}^{-1} C & -B \hat{R}^{-1} B^{T} \\
C^{T} \hat{S}^{-1} C & C^{T} D \hat{R}^{-1} B^{T}-A^{T}
\end{array}\right]
$$

where $\hat{S}=\left(D D^{T}-I\right)$ and $\hat{R}=\left(D^{T} D-I\right)$. Since any purely imaginary scalar $j \omega \in \lambda(\mathcal{M})$ pinpoints a crossover point $\omega$ (in $\mathrm{rad} / \mathrm{s}$ ) of passivity violations, the (possible) nonpassive regions can be accurately located by the imaginary eigenvalue calculation of $\mathcal{M}$. Reference [23] has further developed a half-size singularity matrix for symmetric standard state-space models:

$$
\mathcal{P}=\left(A-B(D-I)^{-1} C\right)\left(B(D+I)^{-1} C-A\right) .
$$

It has been proved that $\omega$ is a crossover point of passivity violations if and only if $\hat{\beta} \in \lambda(\mathcal{P})$ where $\hat{\beta}(\in \mathbb{R})=\omega^{2}>0$. Since $\mathcal{P} \in \mathbb{R}^{n \times n}$ and the eigenvalue computation has $O\left(n^{3}\right)$ complexity, the half-size singularity test is about $8 \times$ faster than the full-size Hamiltonian method. Both of them are reliable but only applicable to standard state-space models. Also, (3) [or (4)] requires $I-D^{T} D$ (or $I-D^{2}$ for symmetric cases) to be nonsingular, which is not guaranteed in all cases.

\section{B. GHM/HGHM Theories for Hybrid DSs}

Denoting the transfer matrix of a hybrid (viz. admittance/impedance) DS by $H(s)$, [2] has proposed the GHM theory: assume $\lambda^{\prime} \notin \lambda\left(\left(D+D^{T}\right) / 2\right)$, then $\left.\lambda^{\prime} \in \lambda\left(\left(H(j \omega)+H^{*}(j \omega)\right) / 2\right)\right)$ if and only if $j \omega \in \lambda(J, K)$. $(J, K)$ is defined as

$$
\begin{aligned}
J & =\left[\begin{array}{cc}
A+B Q^{-1} C & B Q^{-1} B^{T} \\
-C^{T} Q^{-1} C & -C^{T} Q^{-1} B^{T}-A^{T}
\end{array}\right] \\
K & =\left[\begin{array}{rr}
E & \\
& E^{T}
\end{array}\right]
\end{aligned}
$$

where $Q=\left(2 \lambda^{\prime} I-D-D^{T}\right)$. For symmetric DSs, $(J, K)$ reduces to a half-size one

$$
\left(J_{h}, K_{h}\right)=\left(A+B\left(\lambda^{\prime} I-D\right)^{-1} C, E A^{-1} E\right)
$$

whereby the generalized eigenvalue $j \omega$ is replaced by $\beta=\omega^{2}$ accordingly. This is the HGHM theory for hybrid DSs. By setting $\lambda^{\prime}=0$, GHM/HGHM can be used to search the nonpassive regions of hybrid DSs. Due to its half-size nature, HGHM is approximately $8 \times$ faster than GHM [2].

\section{S-GHM AND S-HGHM}

\section{A. S-Parameter GHM (S-GHM) Theory}

Theorem 1: Given the DS (1), $j \omega \notin \lambda(A, E)$ and $\gamma \notin \sigma(D)$, we have $\gamma \in \sigma(S(j \omega))$ if and only if $j \omega \in \lambda(M, N)$, with

$$
\begin{aligned}
M & =\left[\begin{array}{cc}
A-B D^{T} S^{-1} C & -\gamma B R^{-1} B^{T} \\
\gamma C^{T} S^{-1} C & -A^{T}+C^{T} D R^{-1} B^{T}
\end{array}\right] \\
N & =\left[\begin{array}{cc}
E & \\
& E^{T}
\end{array}\right]
\end{aligned}
$$

where $S=D D^{T}-\gamma^{2} I$ and $R=D^{T} D-\gamma^{2} I$. 
Proof: Since the system matrices are real, we have $S^{*}(j \omega)=S^{T}(-j \omega)=B^{T}\left(-j \omega E^{T}-A^{T}\right)^{-1} C^{T}+D^{T}$. Assuming $\gamma \in \sigma(S(j \omega))$ and $\gamma \notin \sigma(D)$, singular value decomposition (SVD) implies that there exist nonzero vectors $v$ and $u$ such that $S(j \omega) u=\gamma v$ and $S^{*}(j \omega) v=\gamma u$, i.e.,

$$
\begin{aligned}
{\left[C(j \omega E-A)^{-1} B+D\right] u } & =\gamma v \\
{\left[B^{T}\left(j \omega E^{T}+A^{T}\right)^{-1}\left(-C^{T}\right)+D^{T}\right] v } & =\gamma u
\end{aligned}
$$

which is equivalent to the compact matrix form

$$
\left[\begin{array}{ll}
C & \\
& B^{T}
\end{array}\right] \Omega_{\omega}^{-1}\left[\begin{array}{ll}
B & \\
& -C^{T}
\end{array}\right]\left[\begin{array}{l}
u \\
v
\end{array}\right]=\left[\begin{array}{cc}
-D & \gamma I \\
\gamma I & -D^{T}
\end{array}\right]\left[\begin{array}{l}
u \\
v
\end{array}\right]_{(8)}
$$

Here $\left[\begin{array}{cc}j \omega E-A & j \omega E^{T}+A^{T}\end{array}\right]$ has been denoted by $\Omega_{\omega}$. For simplicity, we further denote

$$
z:=\Omega_{\omega}^{-1}\left[\begin{array}{ll}
B & \\
& -C^{T}
\end{array}\right]\left[\begin{array}{l}
u \\
v
\end{array}\right] .
$$

Because $\gamma$ is not a singular value of $D$, we can prove that $\left[\begin{array}{cc}-D & \gamma I \\ \gamma I & -D^{T}\end{array}\right]$ is a nonsingular matrix, thus no nonzero vectors are contained in its null space. Since neither $u$ nor $v$ is zero, the right-hand side of (8) is nonzero, which further implies $z$ is a nonzero vector because it is a factor of the left-hand side of (8). Premultiplying both sides of (8) by $\left[\begin{array}{cc}B & \\ & -C^{T}\end{array}\right]\left[\begin{array}{cc}-D & \gamma I \\ \gamma I & -D^{T}\end{array}\right]^{-1}$ would lead to

$$
\left[\begin{array}{cc}
B & \\
& -C^{T}
\end{array}\right]\left[\begin{array}{cc}
-D & \gamma I \\
\gamma I & -D^{T}
\end{array}\right]^{-1}\left[\begin{array}{ll}
C & \\
& B^{T}
\end{array}\right] z=\Omega_{\omega} z .
$$

Because

$M-j \omega N=\left[\begin{array}{ll}B & \\ & -C^{T}\end{array}\right]\left[\begin{array}{cc}-D & \gamma I \\ \gamma I & -D^{T}\end{array}\right]^{-1}\left[\begin{array}{ll}C & \\ & B^{T}\end{array}\right]-\Omega_{\omega}$

(10) can be rewritten as

$$
M z=j \omega N z .
$$

Therefore, $j \omega$ is a purely imaginary generalized eigenvalue of the matrix pencil $(M, N)$. To prove the converse, we define

$$
z^{\prime}=\left[\begin{array}{cc}
-D & \gamma I \\
\gamma I & -D^{T}
\end{array}\right]^{-1}\left[\begin{array}{ll}
C & \\
& B^{T}
\end{array}\right] z=\left[\begin{array}{l}
u^{\prime} \\
v^{\prime}
\end{array}\right]
$$

which is a factor for the left-hand side of (10). Here $u^{\prime}, v^{\prime} \in \mathbb{C}^{n}$. Because $\Omega_{\omega}$ is nonsingular and $z \neq 0$, the right-hand side of (10) and thus $z^{\prime}$ should be nonzero vectors. Premultiplying (10) by $\left[\begin{array}{cc}C & \\ & B^{T}\end{array}\right] \Omega_{\omega}^{-1}$ would lead to

$$
\left[\begin{array}{ll}
C & \\
& B^{T}
\end{array}\right] \Omega_{\omega}^{-1}\left[\begin{array}{ll}
B & \\
& -C^{T}
\end{array}\right] z^{\prime}=\left[\begin{array}{cc}
-D & \gamma I \\
\gamma I & -D^{T}
\end{array}\right] z^{\prime} .
$$

From (13), we can get $S(j \omega) u^{\prime}=\gamma v^{\prime}$ and $S^{*}(j \omega) v^{\prime}=\gamma u^{\prime}$. Because neither $u^{\prime}$ nor $v^{\prime}$ is zero (otherwise both of them and thus $z^{\prime}$ should be zero vectors), $\gamma$ is a singular value of $S(j \omega)$.

\section{B. S-Parameter HGHM (S-HGHM) for Symmetric DSs}

Theorem 2: Assume the DS in (1) is symmetric [i.e., $\left.S^{T}(j \omega)=S(j \omega)\right], j \omega \notin \lambda(A, E)$ and $\gamma \notin D$, we have $\gamma \in \sigma(S(j \omega))$ if and only if $\beta=\omega^{2} \in \lambda\left(M_{h}, N_{h}\right)$, with

$$
\left(M_{h}, N_{h}\right)=\left(X+Y, E(Y-X)^{-1} E\right)
$$

where $X$ and $Y$ are defined as

$$
X=A-B D S^{-1} C, \quad Y=-\gamma B R^{-1} C .
$$

Proof: If the transfer matrix of (1) is symmetric, we have

$$
S^{*}(j \omega)=S(-j \omega)=C(j \omega E+A)^{-1}(-B)+D .
$$

Consequently, $E^{T}, A^{T}, B^{T}, C^{T}$, and $D^{T}$ in (7) can be replaced by $E, A, C, B$, and $D$, respectively. The following nonsingular congruence transform on $(M, N)$ gives a new matrix pencil

$$
\begin{aligned}
\left(M^{\prime}, N^{\prime}\right) & =\left[\begin{array}{cc}
I & I \\
I & -I
\end{array}\right](M, N)\left[\begin{array}{cc}
I & I \\
I & -I
\end{array}\right] \\
& =\left(\left[\begin{array}{ll}
2 X+2 Y & 2 X-2 Y
\end{array}\right],\left[\begin{array}{ll}
2 E & \\
& 2 E
\end{array}\right]\right) .
\end{aligned}
$$

Because the above nonsingular congruence transform does not change the generalized eigenvalues, $j \omega \in \lambda\left(M^{\prime}, N^{\prime}\right)$ if and only if $j \omega \in \lambda(M, N)$. On the other hand, we have proved the equivalence of $j \omega \in \lambda(M, N)$ with $\gamma \in \sigma(S(j \omega))$ in Section III-A, therefore, if $\gamma \in \sigma(S(j \omega))$ we also have $j \omega \in$ $\lambda\left(M^{\prime}, N^{\prime}\right)$, i.e.,

$$
\left[\begin{array}{cc}
-j \omega E & X-Y \\
X+Y & -j \omega E
\end{array}\right]\left[\begin{array}{l}
w_{1} \\
w_{2}
\end{array}\right]=0, \quad\left[\begin{array}{l}
w_{1} \\
w_{2}
\end{array}\right] \neq 0 .
$$

Assuming $X-Y$ being invertible, we get $w_{2}=j \omega(X-$ $Y)^{-1} E w_{1}$ from the upper part of (18). Here, $w_{1}$ is nonzero (otherwise $w_{2}$ is also zero). By virtue of this, (18) reduces to

$$
\left[M_{h}-\omega^{2} N_{h}\right] w_{1}=0 .
$$

Therefore, $\beta=\omega^{2} \in \lambda\left(M_{h}, N_{h}\right)$ if $\gamma \in S(j \omega)$. Analogous to S-GHM, the converse can also be proved. Setting $\omega_{2}=$ $j \omega(X-Y)^{-1} E \omega_{1}$ we can reach (18) from (19). And then using $\lambda(M, N)=\lambda\left(M^{\prime}, N^{\prime}\right)$ we can return to Theorem 1 .

Note that $S$-parameter HGHM only requires the DS transfer matrix $S(j \omega)$ to be symmetric, and it does not pose any restrictions on the symmetry of the system matrices.

\section{PAssivity Test OF DSS}

\section{A. Passivity Test of S-Parameter DSs}

In passivity test, we are interested in the special case of $\gamma=$ 1 , which represents the boundary of passivity violations of an $S$-parameter DS. By setting $\gamma=1$, from S-GHM [in (7)] we get a passivity test matrix pencil $(M, N)=\left(M_{0}, N_{0}\right)$ for scattering DSs with

$$
M_{0}=\mathcal{M}, \quad N_{0}=N .
$$


(a)

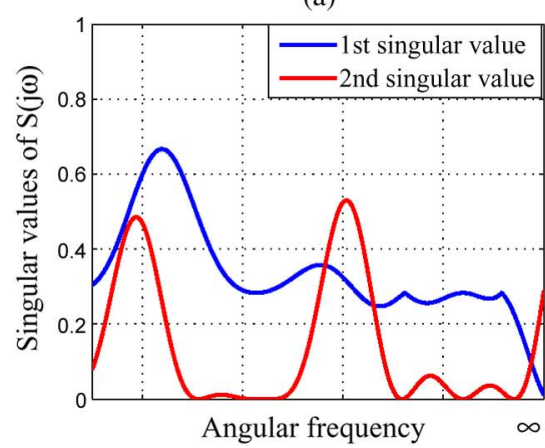

(b)

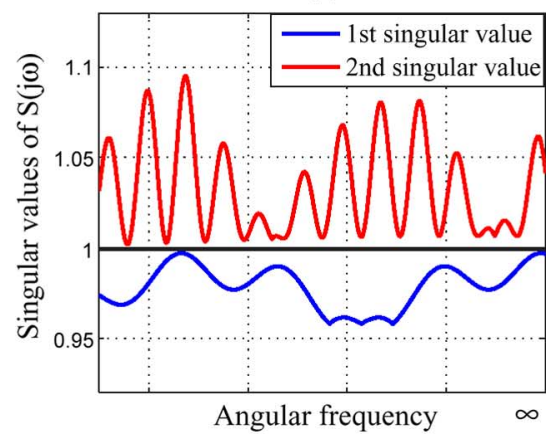

(c)

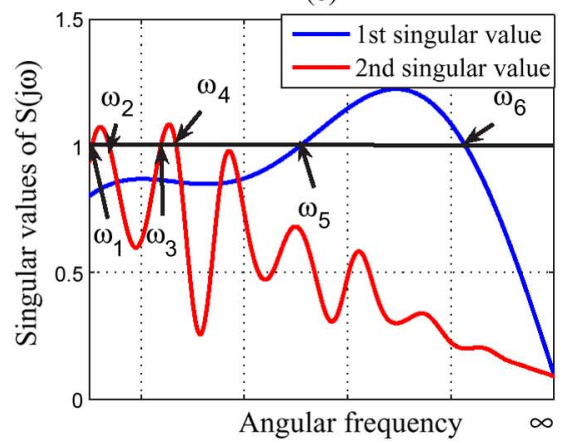

Fig. 1. Illustrative examples for different kinds of DSs. (a) A globally strictly passive DS. This DS has no crossover points and its transfer matrix is always unit-bounded. (b) A consistently nonpassive DS. This DS does not have any crossover points, but it is nonpassive at any frequency point. (c) A DS with locally passive and nonpassive regions. This DS is nonpassive in intervals $l_{2}=\left(\omega_{1}, \omega_{2}\right), l_{4}=\left(\omega_{3}, \omega_{4}\right)$, and $l_{6}=\left(\omega_{5}, \omega_{6}\right)$.

Here, $\mathcal{M}$ is the Hamiltonian matrix defined in (3). Analogously, for symmetric cases, from S-HGHM [in (14)] one can get a halfsize passivity test matrix pencil $\left(M_{h 0}, N_{h 0}\right)$ defined as

$$
\begin{aligned}
M_{h 0} & =A-B(D-I)^{-1} C \\
N_{h 0} & =E\left[\left(B(D+I)^{-1} C-A\right]^{-1} E .\right.
\end{aligned}
$$

If the matrix pencil $\left(M_{0}, N_{0}\right)$ (or $\left(M_{h 0}, N_{h 0}\right)$ for symmetric DSs) has any purely imaginary (or positive real) generalized eigenvalue $j \omega$ (or $\beta=\omega^{2}$ ), then $\omega$ is a crossover frequency point where passivity violation happens.

Note that the scattering DS of interest may still be nonpassive even though no crossover points are computed via S-GHM or S-HGHM. To further identify the possible nonpassive regions, the following procedures could be used.

1) If no crossover points are found, check $S\left(j \omega_{0}\right)$ at an arbitrarily selected frequency point $\omega_{0}$. If $\left\|S\left(j \omega_{0}\right)\right\|<1$, the DS is globally strictly passive [as illustrated in Fig. 1(a)]. Otherwise, if $\left\|S\left(j \omega_{0}\right)\right\|>1$ the DS is nonpassive at any frequency point [as shown in Fig. 1(b)].

2) If $\mathrm{S}-\mathrm{GHM} / \mathrm{S}-\mathrm{HGHM}$ test produces $p$ crossover points $\omega_{1}, \omega_{2}, \ldots, \omega_{p}$ which are increasingly ordered, one can select $p+1$ sampling points $\tilde{\omega}_{k}(k=1,2, \ldots, p+1)$ such that $\tilde{\omega}_{k} \in \ell_{k}$ where $\ell_{1}=\left(0, \omega_{1}\right), \ell_{i}=\left(\omega_{i-1}, \omega_{i}\right)$ for $i=2, \ldots, p$ and $\ell_{p+1}=\left(\omega_{p}, \infty\right)$. If $\left\|S\left(j \tilde{\omega}_{k}\right)\right\|<1$, the DS is passive in the interval $\ell_{k}$; otherwise, it is nonpassive in $\ell_{k}$. An illustrative example is given in Fig. 1(c).

If the DS in (1) is a regular system (when $E$ is invertible), it can be converted to a standard state-space model with $E=I$. Subsequently, for a regular system, the generalized eigenvalue solution in the test pencil $\left(M_{0}, N_{0}\right)$ can be replaced by the standard eigenvalue computation of $M_{0}=\mathcal{M}$. This is in fact the Hamiltonian passivity verification (in (3) of Section II-A). And for symmetric regular systems, substituting $E=I$ into the half-size test pencil in (21), the generalized eigenvalue problem reduces to the standard eigenvalue computation of $\mathcal{P}$ [defined in (4)]. Therefore, the Hamiltonian method and the half-size singularity test in (3) and (4) are the subsets (or special cases) of S-GHM and S-HGHM, respectively.

\section{B. Passivity Test of Hybrid DSs}

A linear LTI system with a hybrid transfer matrix is (strictly) passive if and only if its transfer matrix $H(s)$ is (strictly) positive real [25]:

1) $H(s)$ has no poles in $\operatorname{Re}(s)>0$;

2) $H(s)$ is real for all positive real $s$;

3) $H(s)+H^{*}(s) \geq 0$ (> for strict positive realness) for all $\operatorname{Re}(s)>0$.

Given a square transfer matrix $H(s)$ with $\operatorname{det}(I+H(s)) \neq 0$ for all $\operatorname{Re}(s)>0$, its Moebius-transformed function is [34]

$$
G(s)=(I-H(s))(I+H(s))^{-1} .
$$

If $I+D$ is invertible, a realization of $G(s)=C_{S}\left(s E_{S}-\right.$ $\left.A_{S}\right)^{-1} B_{S}+D_{S}$ is

$$
\begin{aligned}
& E_{S}=E, \quad A_{S}=A-B(I+D)^{-1} C, \quad B_{S}=-\sqrt{2} B(I+D)^{-1} \\
& C_{S}=\sqrt{2}(I+D)^{-1} C, \quad D_{S}=(I-D)(I+D)^{-1}
\end{aligned}
$$

Conversely, $H(s)$ is also a Moebius-transformed function of $G(s)$. For Moebius transformation, the following properties hold [34].

1) Let $H(s)=C(s E-A)^{-1} B+D$ be a positive real DS transfer matrix with nonsingular $I+D$, then its Moebiustransformed function $G(s)$ is bounded real.

2) Given a bounded real DS transfer matrix $G(s)=$ $C_{S}\left(s E_{S}-A_{S}\right)^{-1} B_{S}+D_{S}$ with $I+D_{S}$ being nonsingular and $\operatorname{det}(I+G(s)) \neq 0$ for all $\operatorname{Re}(s)>0$, its Moebius-transformed function $H(s)$ is positive real.

Since $D$ usually has a much lower dimension than $E$ and $A$, the above transformation is very cheap. If we see $G(s)$ as a scattering system, then $H(s)$ is passive if and only if $G(s)$ is passive (i.e., bounded real). Therefore, we can test the passivity of $H(s)$ by checking the bounded realness of $G(s)$ using S-GHM. Further, we note that

$$
G(s)=2(I+H(s))^{-1}-I
$$

which implies that $G(s)$ is symmetric if and only if $H(s)$ is symmetric. Therefore, S-HGHM can also be used for symmetric hybrid cases after Moebius transformation. 
TABLE I

APPLICABILITY OF DIFFERENT PASSIVITY TESTS

\begin{tabular}{|c|c|c|c|c|}
\hline System model & S-GHM & S-HGHM & $\begin{array}{c}\text { Hamiltonian } \\
\text { method }\end{array}$ & $\begin{array}{c}\text { Half-size } \\
\text { singularity }\end{array}$ \\
\hline singular system & yes & yes & no & no \\
\hline regular system & yes & yes & yes & yes \\
\hline asymmetric system & yes & no & yes & no \\
\hline symmetric system & yes & yes & yes & yes \\
\hline
\end{tabular}

\section{Comparison With Traditional Approaches}

Table I compares the applicability of different passivity tests. There are some distinctions among S-GHM/S-HGHM approach with traditional Hamiltonian method and half-size singularity test.

1) S-GHM/S-HGHM can be applied to both singular and regular LTI models, while Hamiltonian method and half-size singularity test are only applicable to regular systems.

2) S-HGHM and half-size singularity test are exclusive to symmetric models, but their full-size counterparts can be used for general cases without symmetric restrictions.

3) S-GHM and S-HGHM search for the passivity violation points via generalized eigenvalue calculation, while traditional methods use standard eigenvalue computation.

4) Due to the half-size nature, S-HGHM and half-size singularity test are $8 \times$ faster than S-GHM and Hamiltonian test, respectively.

5) As will be discussed in Section IV-D, S-GHM/S-HGHM can test the LTI models with $I-D^{T} D$ being singular, whereas the traditional approaches fail to work.

\section{Numerical Issues}

1) The requirement of $1 \notin \sigma(D)$ limits the applications of Hamiltonian method and half-size singularity test. For S-GHM and S-HGHM this restriction can be removed after a small modification called equivalent model conversion [1], [2]. The basic idea is to construct a new DS $S^{\prime}(j \omega)=C^{\prime}\left(j \omega E^{\prime}-A^{\prime}\right)^{-1} B^{\prime}+D^{\prime}$, such that $S^{\prime}(j \omega)=S(j \omega)$ and $1 \notin \sigma\left(D^{\prime}\right) . S^{\prime}(j \omega)$ can be constructed in different ways. In this paper, $S^{\prime}(j \omega)$ is constructed, if necessary, as

$$
\begin{aligned}
& E^{\prime}=\left[\begin{array}{ll}
E & \\
& 0
\end{array}\right], \quad A^{\prime}=\left[\begin{array}{ll}
A & \\
& I
\end{array}\right], \quad B^{\prime}=\left[\begin{array}{c}
B \\
I
\end{array}\right], \\
& C^{\prime}=\left[\begin{array}{ll}
C & \alpha I-D] \quad D^{\prime}=\alpha I
\end{array}\right.
\end{aligned}
$$

with $\alpha \in \mathbb{R}$ and $|\alpha| \neq 1$. Subsequently, the passivity of $S(j \omega)$ can be checked by performing S-GHM/S-HGHM on $S^{\prime}(j \omega)$.

2) With the equivalent model conversion in (25), the restriction of $I+D$ being nonsingular in Moebius transformation can also be removed.

3) In S-HGHM passivity test, $A-B(D+I)^{-1} C$ is assumed to be nonsingular, which is equivalent to $1 \notin \lambda(S(0))$. A proof is given in the Appendix.

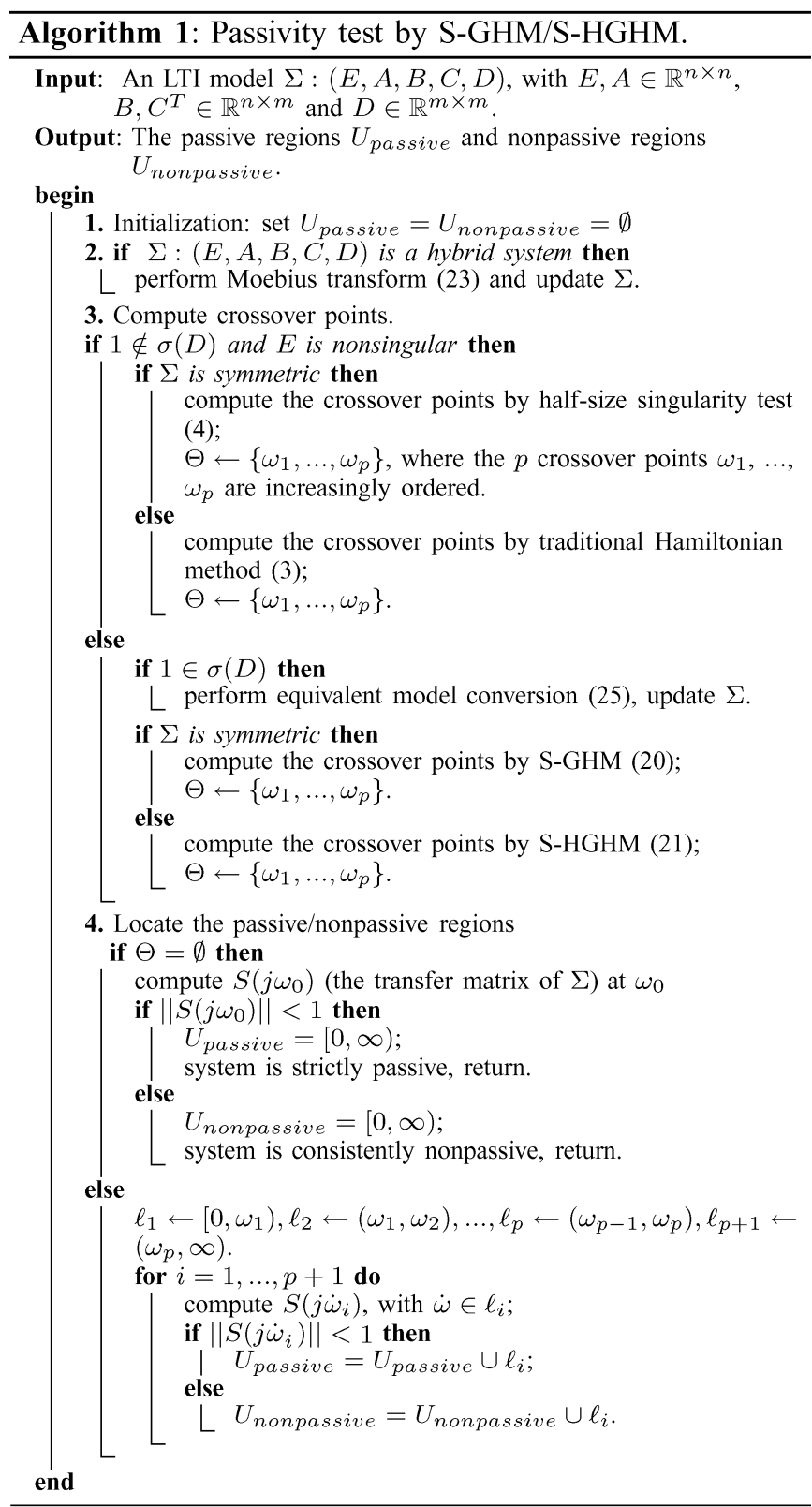

4) The "purely" generalized eigenvalues of $\left(M_{0}, N_{0}\right)$ may appear as conjugate pairs $\lambda_{k}=a_{k} \pm j b_{k}\left(a_{k}, b_{k} \in \mathbb{R}\right)$. Here, $a_{k}$ is the numerical noise induced by the finite machine precision, which is also observed in traditional Hamiltonian method [24]. A small numerical tolerance tol $>0$ can be used to eliminate the noise, and only those solutions with $\left|a_{k}\right|<$ tol are regarded as expected results.

The proposed passivity test is summarized in Algorithm 1.

\section{EXAMPLES}

This section presents some numerical examples to verify the validity and effectiveness of S-GHM/S-HGHM for $S$-parameter and hybrid DSs. All examples are tested in MATLAB R2006a on a $2.66 \mathrm{GHz} 2 \mathrm{G}-\mathrm{RAM}$ PC. 
TABLE II

S-GHM/S-HGHM TEST RESULTS FOR THE ORDER-4 DS

\begin{tabular}{|c|c|c|}
\hline$\lambda\left(M_{0}, N_{0}\right)(j \omega)$ & $\lambda\left(M_{h 0}, N_{h 0}\right)(\beta)$ & $\sqrt{\beta}(\mathrm{rad} / \mathrm{s})$ \\
\hline $5.5 \mathrm{e}-7 \pm j 304491.7$ & 92715196749 & 304491.7 \\
\hline $2.05 \mathrm{e}-14 \pm j 22.86$ & 522.6 & 22.86 \\
\hline
\end{tabular}

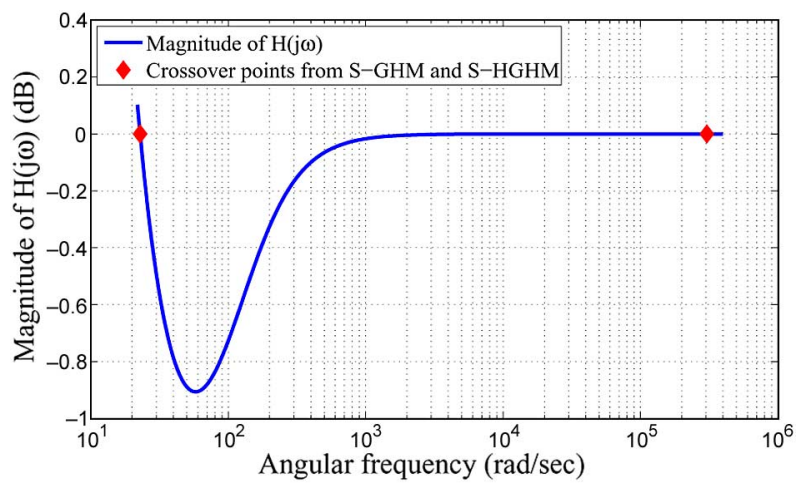

Fig. 2. S-GHM and S-HGHM test results for the order-4 DS model.

\section{A. A Synthetic DS Model}

To show the complete test flow, we consider the bounded realness of the following order-4 single-input single-output (SISO) DS

$$
\begin{aligned}
& E=\left[\begin{array}{llll}
1 & & & \\
& 1 & & \\
& & 0 & 1 \\
& & & 0
\end{array}\right], \quad A=\left[\begin{array}{llll}
-4 & & & \\
& -120 & & \\
& & 1 & \\
& & & 1
\end{array}\right], \\
& B=\left[\begin{array}{c}
1 \\
1 \\
0 \\
10^{-5}
\end{array}\right], \quad C=\left[\begin{array}{llll}
10 & 200 & 10^{-5} & 0
\end{array}\right] \text {, } \\
& D=-1 \text {. }
\end{aligned}
$$

The transfer function of this DS is

$$
S(j \omega)=\frac{10}{j \omega+4}+\frac{200}{j \omega+120}-1+j \omega \times 10^{-10} .
$$

Solving the equation

$$
|S(j \omega)|=1
$$

we get two crossover frequency points: $\omega=22.86 \mathrm{rad} / \mathrm{s}$ and $\omega=304491.7 \mathrm{rad} / \mathrm{s}$. Then we use S-GHM/S-HGHM to compute the crossover points. Note that in this DS $D=-1$, so we need to use equivalent model conversion in advance, which produces an order-5 DS $S^{\prime}(j \omega)$ with $D^{\prime}=0$. Since the transfer function is symmetric, S-HGHM can also be applied for passivity test. The computed results of S-GHM and S-HGHM are listed in Table II. The results of S-GHM and S-HGHM coincide with the solutions to (28), which is illustrated in Fig. 2. Therefore, this scattering DS is bounded real in the angular frequency band of (22.86, 304491.7), and it is nonpassive in the bands of $(0,22.86)$ and $(304491.7, \infty)$.

\section{B. A Symmetric S-Parameter Three-Terminal Filter}

We use a three-terminal filter with symmetric port response to illustrate the application of S-GHM/S-HGHM and the connection to their standard state-space counterparts. The frequency-dependent scattering parameters are measured at 1601 sampling points ranging from $50 \mathrm{MHz}$ to $6 \mathrm{GHz}$. Since it is impulse-free, the input-output response can be described by either a standard state space or a DS. To use the traditional Hamiltonian method and half-size singularity test, we first build an order-120 standard state-space model $(A, B, C, D)$ by vector fitting [15] with 40 common poles. Both the traditional Hamiltonian method [20] and the half-size singularity test [23] show that this model has nine crossover points. The imaginary eigenvalues of $\mathcal{M}$ and the positive real eigenvalues of $\mathcal{P}$ are illustrated in the first and second columns of Table III, respectively. The obtained standard state-space model is then converted to a DS model $H_{d}(j \omega)$ described by $\left(E_{d}, A_{d}, B_{d}, C_{d}, D_{d}\right)$, via the following transformation:

$$
\begin{aligned}
& E_{d}=\left[\begin{array}{ll}
I_{120} & \\
& 0
\end{array}\right], \quad A_{d}=\left[\begin{array}{ll}
A & \\
& I_{3}
\end{array}\right] \\
& B_{d}=\left[\begin{array}{l}
B \\
I_{3}
\end{array}\right], \quad C_{d}=\left[\begin{array}{ll}
C & -D], \quad D_{d}=0 .
\end{array}\right.
\end{aligned}
$$

Then S-GHM and S-HGHM tests on $H_{d}(j \omega)$ also give nine crossover points shown in the third and fourth columns of Table III. Table III shows that the experimental results of all the four methods pinpoint the same boundary frequency points, which coincides with the singular value curves of the transfer matrix in Fig. 3.

\section{A Symmetric Admittance PEEC Reduced Model}

This example is used to show the validity of S-GHM and S-HGHM in the passivity test of admittance or impedance DS models. The original SISO model is an order-480 DS describing a patch antenna structure with admittance parameter as its transfer function, which is obtained by partial element equivalent circuit (PEEC) method [36]. This PEEC model is nonpassive, which may be induced by poor meshing generation, inadequate numerical integration, matrix sparsification or inappropriate geometrical discretization [37]. After model order reduction via PRIMA [13], an order-53 reduced model is obtained, which is nonpassive in the low-frequency band.

Since this model is a hybrid system, S-GHM and S-HGHM can not be directly used. The Moebius-transformed system is first constructed by (23), and then its transfer function $G(s)$ is tested by S-GHM and S-HGHM, respectively. The computed crossover points are listed in Table IV. Fig. 4 shows the magnitude of the Moebius-transformed transfer function equals unity at the computed crossover points. To show the validity, the real part of the original admittance transfer function $H(j \omega)$ is also plotted in Fig. 5, which shows that the proposed S-GHM and 
TABLE III

EXPERIMENTAL RESUlTS OF VARIOUS PASSIVITY TESTS FOR THE THREE-TERMINAL FILTER MODEL

\begin{tabular}{|c|c|c|c|}
\hline $\begin{array}{c}\text { Hamiltonian method: } \\
\text { imaginary results of } \lambda(\mathcal{M})\end{array}$ & $\begin{array}{c}\text { Half-size singularity test: positive real } \\
\text { results of } \lambda(\mathcal{P}) / \text { crossover points }(\mathrm{rad} / \mathrm{s})\end{array}$ & $\begin{array}{c}\text { S-GHM: imaginary } \\
\text { results of } \lambda\left(M_{0}, N_{0}\right)\end{array}$ & $\begin{array}{c}\text { S-HGHM: positive real results of } \\
\lambda\left(M_{h 0}, N_{h 0}\right) / \text { crossover points }(\mathrm{rad} / \mathrm{s})\end{array}$ \\
\hline $6.9 \mathrm{e}-10 \pm j 112253$ & $12600780132 / 112253$ & $2.17 \mathrm{e}-7 \pm j 112253$ & $12600780132 / 112253$ \\
\hline $2.6 \mathrm{e}-10 \pm j 42988.4$ & $18479990052 / 42988.4$ & $2.58 \mathrm{e}-8 \pm j 42988.4$ & $18479990052 / 42988.4$ \\
\hline $1.3 \mathrm{e}-10 \pm j 38173.7$ & $14572294488 / 38173.7$ & $1.06 \mathrm{e}-5 \pm j 38173.7$ & $14572294488 / 38173.7$ \\
\hline $2.2 \mathrm{e}-11 \pm j 34551.3$ & $1193794989.3 / 34551.3$ & $2.93 \mathrm{e}-8 \pm j 34551.3$ & $1193794989.3 / 34551.3$ \\
\hline $4.0 \mathrm{e}-12 \pm j 3109.22$ & $9667288.145 / 3109.22$ & $2.80 \mathrm{e}-6 \pm j 3109.22$ & $9667288.145 / 3109.22$ \\
\hline $2.1 \mathrm{e}-12 \pm j 1631.07$ & $2660378.573 / 1631.07$ & $6.02 \mathrm{e}-7 \pm j 1631.07$ & $2660378.573 / 1631.07$ \\
\hline $3.0 \mathrm{e}-10 \pm j 15871.8$ & $251914404.4 / 15871.8$ & $1.85 \mathrm{e}-8 \pm j 15871.8$ & $251914404.4 / 15871.8$ \\
\hline $8.7 \mathrm{e}-11 \pm j 9879.71$ & $97608669.57 / 9879.71$ & $5.17 \mathrm{e}-7 \pm j 9879.71$ & $97608669.67 / 9879.71$ \\
\hline $8.5 \mathrm{e}-11 \pm j 9895.29$ & $97916796.34 / 9895.29$ & $5.20 \mathrm{e}-7 \pm j 9895.29$ & $97916796.34 / 9895.29$ \\
\hline
\end{tabular}

(a)

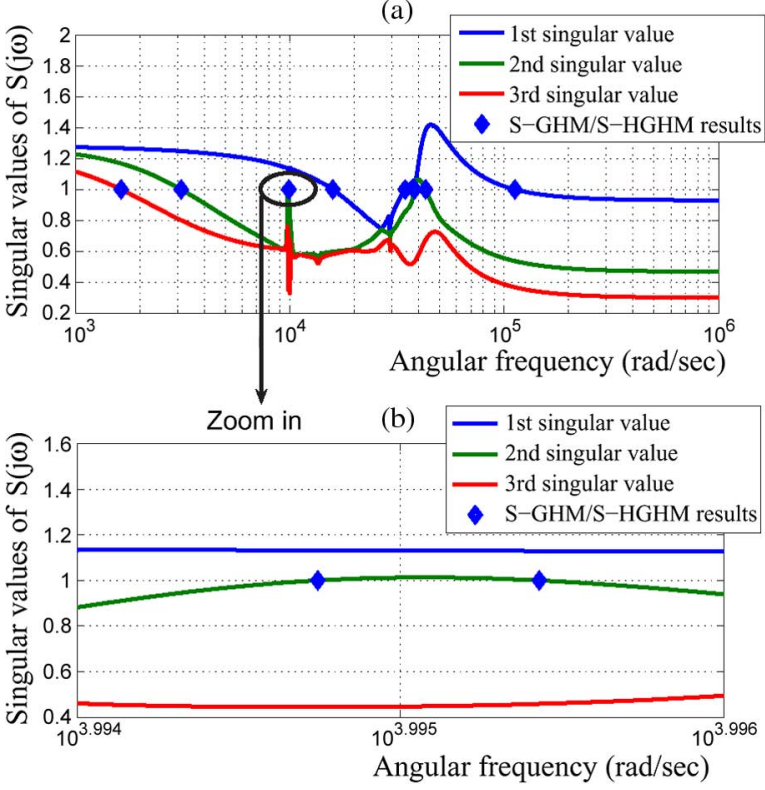

Fig. 3. S-GHM and S-HGHM test results for the three-terminal filter.

TABLE IV

S-GHM AND S-HGHM TEST RESULTS FOR THE ADMITTANCE REDUCED MODEL (ON THE MOEBIUS-TRANSFORMED SYSTEM)

\begin{tabular}{|c|c|c|}
\hline$\lambda\left(M_{0}, N_{0}\right)(j \omega)$ & $\lambda\left(M_{h 0}, N_{h 0}\right)(\beta)$ & $\sqrt{\beta}(\mathrm{rad} / \mathrm{s})$ \\
\hline $1.309 \mathrm{e}-11 \pm j 0.505080$ & 0.255103 & 0.505080 \\
\hline $1.307 \mathrm{e}-11 \pm j 0.505082$ & 0.255110 & 0.505082 \\
\hline $1.127 \mathrm{e}-13 \pm j 1.234402$ & 1.523749 & 1.234402 \\
\hline $3.650 \mathrm{e}-13 \pm j 2.465012$ & 6.076287 & 2.465012 \\
\hline $3.169 \mathrm{e}-13 \pm j 2.560446$ & 6.555922 & 2.560446 \\
\hline $7.587 \mathrm{e}-13 \pm j 4.074095$ & 16.59825 & 4.074095 \\
\hline
\end{tabular}

S-HGHM methods have accurately found the passivity violation points of this hybrid DS.

\section{Multiport DS Model}

This example is used to verify the validity of S-GHM and S-HGHM in general multi-port DS models, and to compare their numerical efficiency. The order-1080 symmetric DS model has 60 ports, and it is built from the measured $S$-parameter data of an electronic system, via the DS-format Loewner matrix fitting [16], [17]. Using S-GHM and S-HGHM tests, 18 crossover points are obtained, which are plotted in Fig. 6. Due to the large amount of crossover points, they are not listed by table. Clearly,

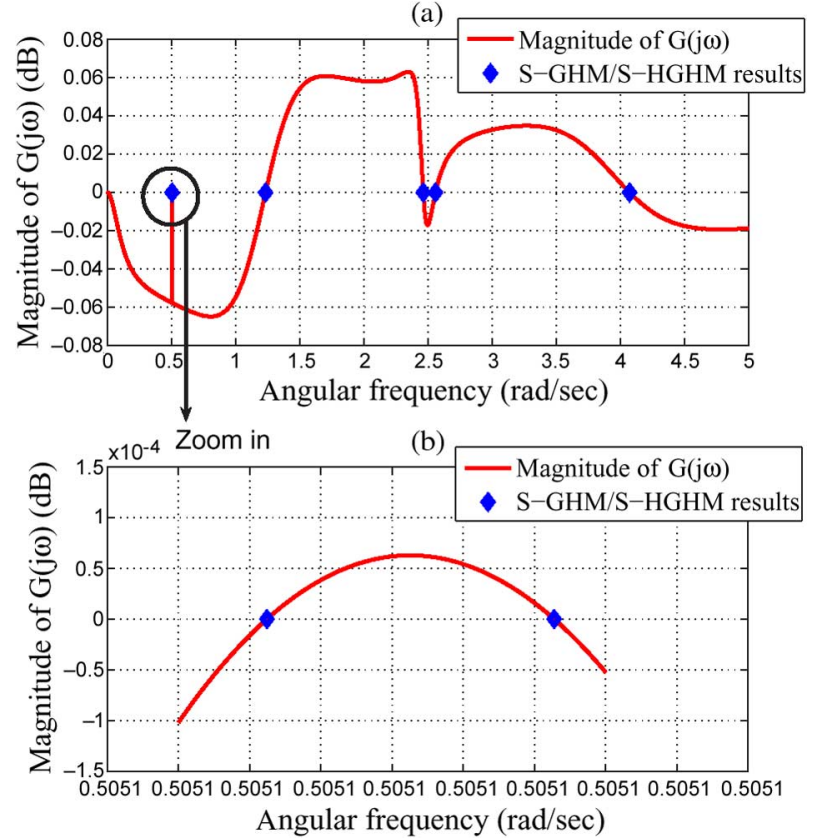

Fig. 4. S-GHM and S-HGHM test results on the Moebius-transformed transfer function of the order-53 admittance reduced model.

Fig. 6(b) shows that the computed results are very accurate. We have listed the CPU times of S-GHM and S-HGHM tests in Table V. It is clear that S-HGHM is about $8 \times$ faster over $\mathrm{S}-\mathrm{GHM}$, which is expected due to its half-size nature and the $O\left(n^{3}\right)$ complexity of generalized eigenvalue calculation.

\section{REMARKS}

1) S-GHM and S-HGHM are purely algebraic passivity verification with similar accuracy to Hamiltonian method and half-size singularity test. Therefore, they are much more reliable and accurate than frequency sweeping methods, which has been verified by their hybrid counterparts [2]. Compared with Hamiltonian method and half-size singularity test, S-GHM and S-HGHM are not restricted by the restriction on $D$ matrix, as verified by the synthetic example.

2) As illustrated in Tables II to IV, the numerical results of S-GHM and Hamiltonian method contain some numerical noise in the real part, which can be eliminated by setting a tolerance. S-HGHM and half-size singularity tests do 


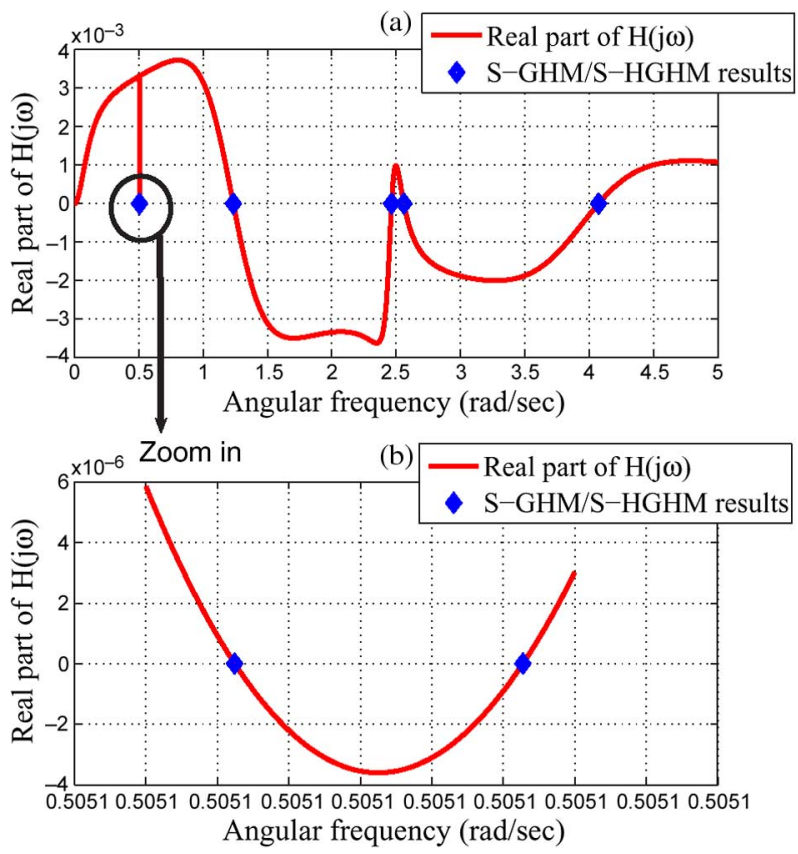

Fig. 5. The real part of the transfer function of the original order-53 admittance DS model. The dots are the results from S-GHM and S-HGHM tests, which are accurately located at the boundaries of passivity violations.
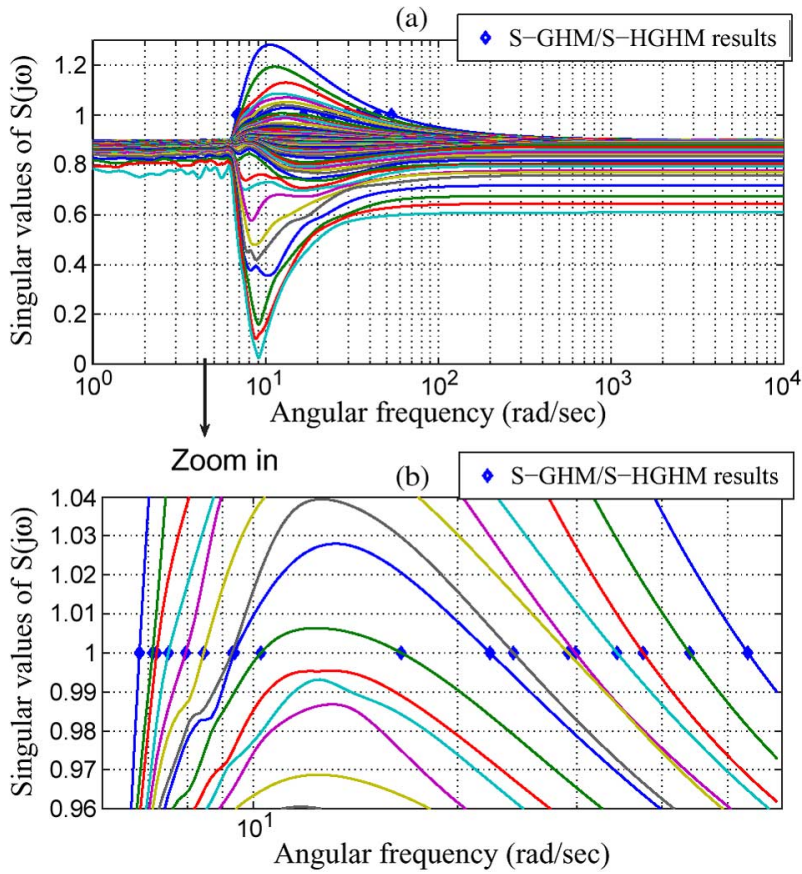

Fig. 6. S-GHM/S-HGHM test results for the multiport $S$-parameter DS model. The dots are the results from S-GHM and S-HGHM tests, which are accurately located at the boundaries of passivity violations.

TABLE V

CPU TIME COMPARISON OF S-GHM AND S-HGHM (IN SECOND)

\begin{tabular}{|c|c|c|c|c|}
\hline Model Size & Port Number & S-GHM & S-HGHM & Speedup \\
\hline 1080 & 60 & 241.2 & 30.45 & 7.92 \\
\hline
\end{tabular}

not suffer from this problem. From the numerical perspective, they are more accurate over the full-size S-GHM and Hamiltonian methods.
3) Due to the half-size nature and the $O\left(n^{3}\right)$ complexity of (generalized) eigenvalue computation, S-HGHM and halfsize singularity test are $8 \times$ faster than their full-size counterparts. This has been verified by the results in [2] and [23] and the CPU timings in Table V. S-GHM and S-HGHM algorithms presented here are based on full-matrix eigensolver, so they are feasible to medium-size (e.g., order1000) physical models. If we consider the Hamiltonian structure of $M_{0}$ and only compute the purely imaginary roots in S-GHM test, the proposed flow is expected to be faster and thus extensible to large and/or sparse DSs. This work has been discussed in [38].

4) In this paper, the passivity at the interval of two adjacent crossover points is identified by the sampling scheme (as shown in Algorithm 1). An alternative approach is to compute the slope signs of the singular value curves at the calculated boundary points, according to the generalized eigenvalue perturbation theory of Hamiltonian matrix pencils. And furthermore, using the perturbation theory, our proposed S-GHM/S-HGHM approach also leads to a DS passivity enforcement scheme. These issues will be reported soon in the future document.

\section{CONCLUSION}

We have extended GHM and HGHM theories to $S$-parameter DSs, which reflect the relationship of the singular values of a DS transfer matrix with its operating frequency. With the proposed methods, the passivity of $S$-parameter and hybrid DSs can be efficiently assessed, and the passive/nonpassive regions can be accurately located. For symmetric DSs, S-HGHM enjoys higher numerical accuracy and an $8 \times$ speedup over S-GHM.

\section{APPENDIX}

In S-HGHM passivity test, the assumption of $A-B(I+$ $D)^{-1} C$ is equivalent to $\operatorname{det}[I-S(j \omega)] \neq 0$ at the DC point (i.e., $\omega=0$ ). The proof is as follows.

Proof: Firstly, we assume that $A-B(I+D)^{-1} C$ is singular, then there exists a vector $p$ such that

$$
\left[A-B(I+D)^{-1} C\right] p=0 \quad p \neq 0 .
$$

Since $A$ is nonsingular, the above equation is equivalent to

$$
A^{-1} B(I+D)^{-1} C p=p .
$$

Denote $(I+D)^{-1} C p$ by $q$, which should be a nonzero vector [otherwise, $p \neq 0$ in (31)]. Premultiplying both sides of (31) by $C$ yields

$$
C A^{-1} B q=(I+D) q, \quad q \neq 0 .
$$

Because $S(0)=D-C A^{-1} B$, (32) can also be written as

$$
[I-S(0)] q=0, \quad q \neq 0
$$

which shows $I-S(0)$ is singular [i.e., $1 \in \lambda(S(0))$ ].

Next, we start with (32) to prove the converse. Since the right side of (32) is nonzero (because $1 \notin \sigma(D)$ and $q \neq 0$ ), we denote $p^{\prime}=A^{-1} B q$ which should also be nonzero. Equation (32) is equivalent to

$$
(I+D)^{-1} C A^{-1} B q=q .
$$


Premultiplying this equation by $A^{-1} B$ we get

$$
A^{-1} B(I+D)^{-1} C p^{\prime}=p^{\prime}, \quad p^{\prime} \neq 0 .
$$

Hence, $A-B(I+D)^{-1} C$ is singular.

\section{REFERENCES}

[1] Z. Zhang, C. U. Lei, and N. Wong, "GHM: A generalized Hamiltonian method for passivity test of impedance/admittance descriptor systems," in Proc. Int. Conf. Comput.-Aided Design, San Jose, CA, Nov. 2009, pp. 767-773.

[2] Z. Zhang and N. Wong, "Passivity test of immittance descriptor systems based on generalized Hamiltonian methods," IEEE Trans. Circuits Syst. II: Express Briefs, vol. 57, no. 1, pp. 61-65, Jan. 2010.

[3] L. Dai, Singular Control Systems, ser. Lecture Notes in Control and Information Sciences 118. Berlin, Germany: Springer-Verlag, 1989.

[4] E. L. Yip and R. F. Sincovec, "Solvability, controllability and observability of continuous descriptor systems," IEEE Trans. Autom. Control, vol. 26, no. 3, pp. 702-707, Jun. 1981.

[5] P. Feldmann and R. W. Freund, "Reduced-order modeling of large linear subcircuits via a block Lanczos algorithms," in Proc. Design Automation Conf., 1995, pp. 474-479.

[6] N. Mi, S. X.-D. Tan, Y. Cai, and X. Hong, "Fast variational analysis of on-chip power grids by stochastic extended Krylov subspace method," IEEE Trans. Computer-Aided Design, vol. 27, no. 11, pp. 1996-2006, Nov. 2008.

[7] B. N. Bond and L. Daniel, "A piecewise-linear moment-matching approach to parameterized model-order reduction for highly nonlinear systems," IEEE Trans. Computer-Aided Design, vol. 26, no. 12, pp. 2116-2129, Dec. 2007.

[8] N. Dong and J. Roychowdhury, "General-purpose nonlinear model order reduction based on piecewise polynomial representations," IEEE Trans. Computer-Aided Design, vol. 27, no. 2, pp. 249-261, Feb. 2008.

[9] I. Balk, "On a passivity of the Arnoldi based model order reduction for full-wave electromagnetic modeling," IEEE Trans. Adv. Packag., vol. 24, no. 3, pp. 304-308, Aug. 2001.

[10] H. Wu and A. C. Cangellaris, "Model-order reduction of finite-element approximations of passive electromagnetic devices including lumped electrical-circuit models," IEEE Trans. Microwave Theory Tech., vol. 52, no. 9, pp. 2305-2313, Sep. 2004.

[11] D. Ioan and G. Ciuprina, "Reduced order models of on-chip passive components and interconnects, workbench and test structures," in Model Order Reduction: Theory, Research Aspects and Applications. Berlin, Germany: Springer, Aug. 2008, pp. 447-467.

[12] T. Stykel, "Gramian based model reduction for descriptor systems," Math. Control, Signals, Syst., vol. 16, no. 4, pp. 297-319, Mar. 2004.

[13] A. Odabasioglu, M. Celik, and L. Pileggi, "PRIMA: Passive and reduced-order interconnect macromodeling algorithm," IEEE Trans. Computer-Aided Design, vol. 17, no. 8, pp. 645-654, Aug. 1998.

[14] N. Marques, M. Kamon, L. Silveira, and J. White, "Generating compact, guaranteed passive reduced-order models of 3-D RLC interconnects," IEEE Trans. Adv. Packag., vol. 27, no. 4, pp. 569-580, Nov. 2004 .

[15] B. Gustavsen and A. Semlyen, "Rational approximation of frequency domain responses by vector fitting," IEEE Trans. Power Del., vol. 143, no. 3, pp. 1052-1061, Jul. 1999.

[16] S. Lefteriu and A. C. Antoulas, "Modeling multi-port systems from frequency response data via tangential interpolation," in IEEE Workshop Signal Propogat. Interconnects, May 2009, pp. 1-4.

[17] A. J. Mayo and A. C. Antoulas, "A framework for the solution of the generalized realization problem," Linear Algebra Appl., vol. 425, no. 2-3, pp. 634-662, Sep. 2007.

[18] S. Grivet-Talocia, "An adaptive sampling technique for passivity characterization and enforcement of large interconnect macromodels," IEEE Trans. Adv. Packag., vol. 31, no. 4, pp. 673-683, Nov. 2008

[19] D. Saraswat, R. Achar, and M. Nakhla, "Fast passivity verification and enforcement via reciprocal systems for interconnects with large order macromodels," IEEE Trans. VLSI Syst., vol. 15, no. 1, pp. 48-59, Jan. 2007.

[20] S. Grivet-Talocia, "Passivity enforcement via perturbation of Hamiltonian matrices," IEEE Trans. Circuits Syst. I, vol. 51, no. 9, pp. 1755-1769, Sep. 2004.

[21] S. Grivet-Talocia and A. Ubolli, "A comparative study of passivity enforcement schemes for linear lumped macomodels," IEEE Trans. Adv. Packag., vol. 31, no. 4, pp. 673-683, Nov. 2008.

[22] D. Saraswat, R. Achar, and M. Nakhla, "Global passivity enforcement algorithms for macromodels of interconnect subnetworks characterized by tabulated data," IEEE Trans. VLSI Syst., vol. 13, no. 7, pp. 819-831, Jul. 2005 .
[23] B. Gustavsen and A. Semlyen, "Fast passivity assessment for $S$-parameter rational models via half-size test matrix," IEEE Trans. Microwave Theory Tech., vol. 56, no. 12, pp. 2701-2708, Dec. 2008.

[24] A. Semlyen and B. Gustavsen, "A half-size singularity test matrix for fast and reliable passivity assessment of rational models," IEEE Trans. Power Del., vol. 24, no. 1, pp. 345-351, Jan. 2009.

[25] R. W. Freund, "An extension of positive real lemma to descriptor systems," Optimizat. Methods Software, vol. 19, no. 1, pp. 69-87, Feb. 2004.

[26] N. Wong and C. K. Chu, "A fast passivity test for stable descriptor systems via skew-Hamiltonian/Hamiltonian matrix pencil transformations," IEEE Trans. Circuits Syst. I, vol. 55, no. 2, pp. 635-643, Mar. 2008.

[27] P. Benner and D. Chu, "A new test for passivity of descriptor systems," Oberwolfach Rep., vol. 2, no. 1, pp. 571-574, Nov. 2005.

[28] D. Chu and R. C. E. Tan, "Algebraic characterizations for positive realness of descriptor systems," SIAM J. Matrix Anal. Appl., vol. 30, no. 1, pp. 197-222, 2008.

[29] L. Zhang, J. Lam, and S. Xu, "On positive realness of descriptor systems," IEEE Trans. Circuits Syst. I, vol. 49, no. 3, pp. 401-407, Mar. 2002.

[30] C. Yang, Q. Zhang, Y. Lin, and L. Zhou, "Positive realness and absolute stability problem of descriptor systems," IEEE Trans. Circuits Syst. I, vol. 54, no. 5, pp. 1142-1149, May 2007.

[31] Y. Liu and N. Wong, "Fast sweeping methods for checking passivity of descriptor systems," in Proc. IEEE Asia Pacific Conf. Circuits Syst., Dec. 2008, pp. 566-569.

[32] N. Wong, "An efficient passivity test for descriptor systems via canonical projector techniques," in Proc. Design Automat. Conf., San Francisco, CA, Jul. 2009, pp. 957-962.

[33] H. S. Wang, C. F. Yung, and F. R. Chang, "Bounded real lemma and $H_{\infty}$ control for descriptor systems," IEE Proc. Control Theory Appl., vol. 145, no. 3, pp. 316-322, 1998.

[34] T. Reis and T. Stykel, Passivity-preserving balanced truncation for electrical circuits 2008 [Online]. Available: http://www.math.tuberlin.de/stykel/

[35] Z. Zhang and N. Wong, "An extension of the generalized Hamiltonian method to $S$-parameter descriptor systems," in Proc. Asia South Pacific Design Autom. Conf., Taipei, Taiwan, Jan. 2010, pp. 43-47.

[36] H. Heeb and A. E. Ruehli, "Three-dimensional interconnect analysis using partial element equivalent circuits," IEEE Trans. Circuits Syst. I, vol. 39, no. 11, pp. 974-982, Nov. 1993.

[37] J. Ekman, G. Antonini, A. Orlandi, and A. E. Ruehli, "Stability of PEEC models with respect to partial element accuracy," in Int. Symp. Electromagn. Compatibil., Aug. 2004, vol. 1, pp. 271-276.

[38] Z. Zhang and N. Wong, "An efficient projector-based passivity test for descriptor systems," IEEE Trans. Computer-Aided Design, accepted for publication.

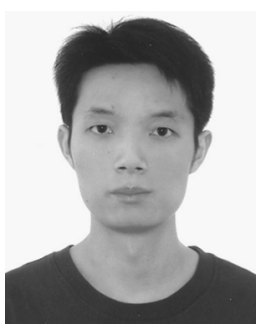

Zheng Zhang (S'09) received the B.Eng degree in electronic science and technology, from Huazhong University of Science and Technology, China, in 2008. He was a visiting graduate student with the Department of Computer Science and Engineering, University of California at San Diego, La Jolla, $\mathrm{CA}$, in 2009. He is an MPhil candidate in electrical and electronic engineering, The University of Hong Kong.

His current research is about computer-aided design of very large-scale integrated circuits (VLSI CAD), with emphasis on numerical methods for model order reduction, analog/RF circuit simulation, power grid simulation, and signal integrity analysis.

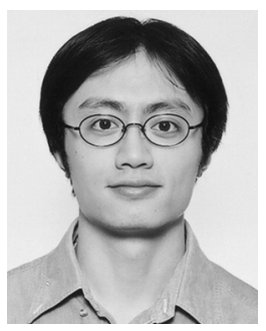

Ngai Wong (S'98-M'02) received the B.Eng. degree (with first class honors) and the Ph.D. degree, both in electrical and electronic engineering, from The University of Hong Kong in 1999 and 2003, respectively.

He was an intern at Motorola Inc., Hong Kong, during 1997-1998, specializing in product testing. He was a visiting scholar at Purdue University, West Lafayette, IN, in 2003. Currently, he is an Assistant Professor at the University of Hong Kong. His research interests include VLSI model order reduction and simulation, digital filter design, sigma-delta modulators, and optimization problems in communication and VLSI applications. 